\title{
Embryo und Grundgesetz. Schutz des Lebens und der Menschenwürde vor Nidation und Geburt.
}

I.

Die schwierige Rolle des Rechts im Geflecht von wissenschaftlichem Fortschritt, technologischer Risikensteuerung, gesellschaftlichen Moralvorstellungen, ökologischen und ökonomischen Interessen lässt sich gegenwärtig auf kaum einem Feld so eindrucksvoll beobachten wie auf dem der Biotechnik und insbesondere bei den offenen Problemen der Reproduktionsmedizin. Die Schwierigkeiten, aber auch die Chancen rechtswissenschaftlicher Bemühungen in - vielleicht - rechtspolitischer Absicht auf diesem Gebiet werden noch deutlicher sichtbar, wenn man den Versuch einer - altmodisch ausgedrückt - rechtsvergleichenden Betrachtung unternimmt, was zwischen verwandten Rechtsordnungen in einem zusammenwachsenden Europa eigentlich alltäglich und selbstverständlich sein sollte. Dabei wird man bald gewahr, dass man bei der vergleichenden Analyse der einfachgesetzlichen Rechtsnormen nicht stehen bleiben kann, dass man den Blick auf die übergeordneten Normen der Verfassung ausweiten und schließlich zu einer kulturvergleichenden Betrachtung gelangen muss.

Nimmt man auf diese Weise das deutsch-spanische Verhältnis zu unserem Thema ins Visier, so springt ins Auge, dass die spanische Rechtsentwicklung früher und weiter gediehen ist als die deutsche, und man wird nach den Ursachen hierfür fragen.

Diese Feststellung gilt zunächst für die einfachgesetzliche Rechtslage.

Spanien hat mit drei Gesetzen einen umfassenden normativen Rahmen für die meisten pränatalen reproduktionsmedizinischen Tätigkeiten und Sachverhalte geschaffen, während in Deutschland das Embryonenschutzgesetz ESchG (vom 13.12.1990, BGBl. I S.2746) und das Stammzellgesetz StZG (vom 28.6.2002, BGB1. I S. 2277) im wesentlichen strafrechtliche Verbote beziehungsweise enggefasste Genehmigungsvoraussetzungen für die ausnahmsweise erlaubnisfähige Forschung mit embryonalen Stammzellen normieren. Der Entwurf eines Gesetzes zur Regelung der Präimplantationsdiagnostik PID ${ }^{1}$, welchen die Liberalen (FDP) im November 2001 eingebracht hatten, ${ }^{2}$ hatte keinerlei Chancen im Parlament und fand sein Ende mit dem der Legislaturperiode. Nicht untypisch für die ,Stimmung، der Mehrheit im Deutschen Bundestag ist der im Januar 2003 von allen Fraktionen mit Ausnahme der FDP gefasste Beschluss, der ein - möglichst weltweites - Verbot des Klonens, des reproduktiven wie des therapeutischen, fordert. Und die soeben (April 2003) publizierte

1 PID = im Angloamerikanischen PGD: Preimplantation Genetic Diagnosis. Zu unterscheiden von der in auch Deutschland zulässigen PND = Pränataldiagnostik, welche die Phase der Schwangerschaft betrifft.

2 Vgl. Bundestags-Drucksache 14/ 7415 vom 9. 11. 2001. 
Stellungnahme des Nationalen Ethikrates, eines 24-köpfigen Gremiums unabhängiger Sachverständiger, zur »Genetischen Diagnostik vor und während der Schwangerschaft«, also zu PID und PND, ist aus zwei Voten zusammengefügt: aus einem die PID strikt ablehnenden Minderheitsvotum und aus einem Mehrheitsvotum, das für eine »verantwortungsvolle, eng begrenzte Zulassung der PID« plädiert. Einigkeit besteht jedoch unter allen Sachverständigen darüber, dass »alle mit der Reproduktionsmedizin zusammenhängenden wesentlichen Fragen in einem speziellen Fortpflanzungsmedizingesetz« geregelt werden sollen. Wenngleich man spanischen Stimmen zustimmen möchte, ${ }^{3}$ die vor einer Überforderung des Rechts warnen, das nicht überall konsensfähige Lösungen der hier offenen Fragen anbieten könne, so verdient doch der spanische Gesetzgeber großen Respekt, der mit den beiden Gesetzen Nr. 35/1988 über die Assistierte menschliche Reproduktion (RCL 1988\2332 Legislación) und Nr. 42/ 1988 über Embryonen (RCL 1988\2619 Legislación) sowie mit der Änderung durch das Organische Gesetz 10/1995 vom 23. 11. 1995 (RCL 1995\3170) für viele in Deutschland noch umstrittene Einzelfragen schon brauchbare Lösungen gefunden hat. Und das Grundsatzurteil des Tribunal Constitucional vom 17. Juni 19994, welches die Verfassungsmäßigkeit des Gesetzes Nr. 35/ 1988 bestätigt hat, hat damit wesentlich zu einer Klärung gerade solcher grundsätzlichen Fragen beigetragen, die das Bundesverfassungsgericht einer abschließenden und befriedenden Antwort noch nicht zuführen konnte.

Vor allem zwei Fragen sind es, deren eindeutige Beantwortung durch die spanische Gesetzgebung und Rechtsprechung diesen nicht nur zu schärferen rechtssystematischen und begrifflichen Konturen, sondern der ganzen Diskussion auch zu einer erfreulichen Entmetaphysizierung verholfen hat, von der man hierzulande noch weit entfernt ist.

Die erste betrifft die Anerkennung der Nidation als eine entscheidende Zäsur, also den Zeitpunkt etwa vierzehn Tage nach der Befruchtung und Kernverschmelzung. Von diesem Moment an ist das neue Leben dauerhaft im Uterus eingepflanzt und begründet eine »unmittelbare, abhängige und vitale Beziehung zu der Schwangeren«. Von diesem Augenblick an spricht man in Spanien von einem »Embryo«, und nach dessen Organbildung von »Fetus «. ${ }^{5}$ Das Gesetz Nr. 42/ 1988 handelt demgemäß nur von »Embryonen « und »Feten«, mithin von der Phase der Schwangerschaft. ${ }^{6}$ Die Gestalt des Lebens vor der Nidation, extrakorporal (in vitro) oder intrakorporal wird als »Präembryo« (»preembrión«) oder »präimplantativer Embryo« ( »embrión preimplantatorio«) bezeichnet; ausschließlich dieser ist Gegenstand des Gesetzes Nr. 35/ 1988 zur Regelung der Techniken der Assistierten Reproduktion.

Wenn man die heftige und keineswegs immer fruchtbare deutsche Diskussion über den Zeitpunkt des Beginns »menschlichen Lebens« oder aber »des Lebens eines Men-

3 Vgl. etwa Carlos Lema Añón, Los problemas pendientes de la regulación jurídica española sobre reproducción humana asistida:.., parte II, Rev Der Gen H 13/2000 103 ff., 118.

4 1999/ 11251 TC Pleno, S 17. 6. 1999, Fecha BOE 08-07-1999.

5 S. Jefatura del Estado, 1988/29681 Ley 42/1988, BOE 314/1988, pág. 36766, Exposicion de motivos.

6 So deutlich auch BVerfGE 39, 1, 37 und BVerfGE 88, 203, 251. 
schen « verfolgt hat, die sich vielfach auf die beiden Leitentscheidungen des Bundesverfassungsgerichts zur Abtreibung ${ }^{7}$ beruft, dann kann man bei der Lektüre der allgemeinen Begründung zum Reproduktions-Gesetz Nr. 35/ 1988 (sub. II.) eine gewisse Befriedigung nicht unterdrücken, insofern der spanische Gesetzgeber zur argumentativen Untermauerung seiner Unterscheidung von Embryo und Präembryo neben anderen gewichtigen Stimmen und neben der Entscheidung 11/4/85 (RTC 1985153) des Tribunal Constitucional auch auf die Entscheidung des Bundesverfassungsgerichts vom 25. Februar 1975 rekurriert. Dem Augenblick der Einnistung komme notwendig eine biologische Bewertung zu, denn vorher »bewege sich die embryonale Entwicklung in der Ungewissheit «. ${ }^{8}$

Die zweite Frage, von der ebenfalls sogleich noch die Rede sein wird, betrifft das Verhältnis von Lebensschutz und Menschenwürdeschutz und damit die verfassungsrechtliche Lage. Während das Recht auf Leben in Art. 15 der Spanischen Verfassung unter der Überschrift»von den Grundrechten und öffentlichen Freiheiten« im 1. Abschnitt des 2. Kapitels des Titels I als ein echtes Grundrecht normiert wird, dessen gesetzliche Ausgestaltung dem besonderen Gesetzesvorbehalt für ein »Organisches Gesetz« (Art. 81 Abs.1 SpanVerf.) unterliegt, gilt dies für die in Art. 10 angesprochene Personwürde (dignidad de la persona) nicht, weil sie von der Verfassung (»objektivrechtlich«) als »Fundament der politischen Ordnung und des sozialen Friedens « gesehen wird. So konnte das Gericht die Rüge eines Verstoßes gegen das Erfordernis eines »Organischen Gesetzes « zurückweisen, weil auch die Regelung der Reproduktionstechniken in der Präembryonal-Phase nicht in den Schutzbereich des Rechtes auf Leben gemäß Art. 15 SpanVerf. falle. ${ }^{9}$ Von einer so engen Verbindung von Würdeschutz und Lebensschutz wie in der deutschen Rechtsprechung - dazu im Folgenden - kann demnach im spanischen Verfassungsrecht nicht die Rede sein. Das Verständnis der »Würde des Menschen « (la dignidad de la persona) als ein objektives Grundprinzip der Verfassungsordnung - Art. 10, 1. SpanVerf. - und nicht, wie nach Art. 1 Abs. 1 Grundgesetz - als ein (auch) subjektives Grundrecht, entlastet die Diskussion von ideologischen Aufladungen, denen man kaum entrinnen kann, wenn man bereits den Gameten oder Blastozysten in der Petrischale Rechtssubjektivität zuerkennt.

\section{II.}

Im November und Dezember 1991 führte der Sonderausschuss des Deutschen Bundestages fünf Tage lang Anhörungen zu den schwierigen und heiß umstrittenen, mit der Neuregelung des Abtreibungsrechts zusammenhängenden Fragen durch. Bis es spät in der Nacht des 25. Juni 1992 zur Schlussabstimmung im Plenum des Parlaments kam, hatte der Sonderausschuss die vorliegenden Gesetzesentwürfe in insgesamt

7 Siehe vorige Note.

8 S. Jefatura del Estado, Ley 35/ 1988, vom 22. November, RCL 198812332 Legislación, BOE 24. 11. 1988, Nr. 282/1988, pág. 33373.

9 Urteil des TC Pleno vom 17. 6. 1999, (s. N. 4), sub Fundamentos de derecho, CUARTO. 
zwanzig Sitzungen beraten. Obwohl das »Schwangeren- und Familienhilfegesetz« vom 27. Juli 1992 mit 357 JA- gegen 284 NEIN-Stimmen bei 16 Enthaltungen eine deutliche Mehrheit fand, brachten die Bayerische Staatsregierung und 249 Bundestagsabgeordnete es auf den Prüfstand in Karlsruhe. Das nach zweitägiger mündlicher Verhandlung im Dezember 1992 schließlich am 28. Mai 1993 verkündete Urteils des Bundesverfassungsgerichts umfasst 163 Druckseiten, die Entscheidungsformel allein knapp sechs. ${ }^{10}$ Diese Zahlen mögen anschaulich machen, dass sich wahrlich niemand eine Entscheidung zum Thema »Schwangerschaftsunterbrechung « leicht gemacht hat. Dennoch ist der Streit noch längst nicht völlig beigelegt, ist der »Rechtsfriede « nur unvollkommen hergestellt; die Gegensätze schwelen weiter. Zwar hört man hierzulande, anders als in den USA, nicht davon, dass Ärzte beim Verlassen ihrer Abtreibungskliniken erschossen werden. Doch musste der Bundesgerichtshof erst vor kurzem über die Zulässigkeit des Verteilens von Flugblättern vor einem Klinikum urteilen, in welchen vom »Kinder-Mord im Mutterschoß auf dem Gelände des Klinikums N.« und von »damals: Holocaust, heute: Babycaust «- die Rede war. ${ }^{11}$ Offenbar gibt es Streitfragen, in denen sich Auffassungen buchstäblich »unversöhnlich « gegenüberstehen und trotz aller Diskursbemühungen auch nicht zu einem Ausgleich kommen. Man hat solche Situationen als »desintegrative Identitätskonflikte« - im Unterschied zu prinzipiell lösbaren bloßen »Verteilungskonflikten« - beschrieben, um anzudeuten, dass sie der sozialen wie der normativen Integration eines Gemeinwesens besondere Schwierigkeiten bereiten. ${ }^{12}$ Die Fragen, wann das menschliche Leben und deshalb sein rechtlicher Schutz beginne bzw. beginnen müsse, oder die Frage nach dem Beginn (und Ende) der Trägerschaft der unantastbaren Menschenwürde und die Frage, welche Tatbestände in welchem Stadium des Menschseins als Verletzungen, »Antastungen «, der Menschenwürde zu qualifizieren sind, gehören möglicherweise zu jener Kategorie schwer auflösbarer »Identitätskonflikte«. Ihre Erscheinungsformen belehren den Juristen immer wieder über die Zerbrechlichkeit jener Synthese aus gewissensgeprüfter Subjektivität und institutionell-autoritativer Inanspruchnahme objektiver Geltung, welche die notwendige Basis einer normativ wirksam integrierenden Rechtsordnung bildet. Diese Synthese kann nach beiden Seiten hin verfehlt werden, so hinsichtlich der Anerkennungsleistung des Subjekts, wenn etwa ein fundamentalistischer Kernkraftgegner die Bezahlung seiner Stromrechnung mit Hinweis auf den nuklearen Ursprung der genutzten Elektrizität verweigert oder wenn ein Zeuge Jehovas nicht nur den Kriegs- oder Zivildienst, sondern auch jegliche Steuerzahlung verweigert, weil er den Staat insgesamt als »Bestandteil der Welt Satans« ansieht. $^{13}$

Nach der anderen Seite hin wird die Synthese verfehlt, wenn etwa Gerichte die Menschenwürde als einen »objektiven, unverfügbaren Wert«, der aber als solcher der

10 Vgl. BVerfGE 88, 203 ff. Zum Verfahren E. Denninger/W. Hassemer, KritV 1/1993, 78 ff.

11 BGH Urteil vom 30. 5. 2000, VersR 2000, 1162; dazu S. Dulitz in JA 2/ 2001, 89 ff.

12 Vgl. G. Frankenberg, Tocquevilles Frage, in: Schuppert/Bumke (Hrsg.), Bundesverfassungsgericht und gesellschaftlicher Grundkonsens, 2000, S. 31; Denninger, Normalfall oder Grenzfall als Ausgangspunkt rechtsphilosophischer Konstruktion ? in KritV 1/2002, 5 ff.

13 Vgl. das Zitat in BVerfGE 102, 370, 397. 
richterlichen Erkenntnis zugänglich sei, bezeichnen und deshalb das freiwillige Auftreten einer Frau in einer »Peep-Show « oder den sogenannten »Zwergenweitwurf« als Verletzung der Menschenwürde verbieten. ${ }^{14}$ Indem man den verfehlenden richterlichen Objektivitätsanspruch als Attitüde der Macht durchschaut, wird man zugleich mit der Frage nach möglichen Grenzen staatlicher Regulierungsmacht überhaupt in Fragen notwendiger Gewissensentscheidung konfrontiert. Der Philosoph hat es hier leichter, Enthaltsamkeit zu fordern, etwa so: »Der weltanschaulich neutrale Staat kann, wenn er demokratisch verfasst ist und inklusiv verfährt, in einer »ethisch « umstrittenen Inanspruchnahme von Artikel 1 und 2 GG nicht Partei ergreifen. Wenn die Frage des Umgangs mit ungeborenem menschlichem Leben einen ethischen Charakter hat, muss vernünftigerweise mit einem begründeten Dissens, wie er sich in der Bundestagsdebatte vom 31. Mai 2001 abgezeichnet hat, gerechnet werden. $\ll^{15}$ Freilich: Der als solcher angerufene Verfassungsrichter kann dem Konflikt zwischen kollidierenden Schutzgütern nicht ausweichen, er muss Stellung nehmen.

In solchen Auseinandersetzungen, und dies bestätigt der gegenwärtige Streit über die Zulässigkeit des forschenden oder therapeutischen Umgangs mit Embryonen in vitro, also vor ihrer Einpflanzung in den Uterus, vor der »Nidation «, beruft sich jede Streitpartei auf jeweils ihre Interpretation der Verfassung als die angeblich einzig richtige, hier etwa auf den Begriff der »Würde des Menschen« in Art. 1 Abs.1 GG und den Begriff des »Lebens« und des »Rechts auf Leben« des Art. 2 Abs.2 GG. Großformatige Zeitungsanzeigen wie »Stoppt PID \& Klonen « zeugen zwar vom Engagement und der Finanzkraft der Auftraggeber, nicht aber von der Stärke ihrer Argumente. ${ }^{16}$ Nicht annoncieren sondern argumentieren ist angesagt.

Dabei möchte ich zunächst nachdrücklich davor warnen, übertriebene Erwartungen an die geschriebene Verfassung zu richten, Erwartungen, die sie nicht erfüllen kann, weder hinsichtlich ihres deskriptiven, feststellenden noch hinsichtlich ihres präskriptiven, normativen Gehalts. Die Verfassung hält nicht auf alle Fragen eine eindeutige und fertige Antwort parat, die gewissermaßen jedermann bei einiger Sorgfalt im Umgang mit dem Text mit demselben Ergebnis wie ein anderer herauszufinden vermöchte. Vielmehr verwendet sie an zentralen Stellen Begriffe, die man als Grenzbegriffe bezeichnen kann, weil sie die Grenzen rein rechtlich immanenter Definitionsmacht überschreiten und auf undefinierte außerrechtliche Lebensbereiche hinweisen, die aber doch zur Anknüpfung rechtlicher Folgen einer »juristischen Definition « bedürfen. Beispiele hierfür bieten etwa die Begriffe »Religion «, »Gewissen« oder »Kunst«. Wenn bei einer möglicherweise beleidigenden Karikatur der Konflikt »Ehrenschutz« vs. »Kunstfreiheit « zu entscheiden ist, dann muss der Richter Kriterien dafür angeben können, ob er ein »Kunstwerk « vor sich hat oder nicht, d.h. er muss »Kunst« definieren, ohne doch dabei in »Kunstrichtertum « zu verfallen. ${ }^{17}$ In besonde-

14 Einer Notiz in der F.A.Z. vom 30. 11. 2001, S. 14, zufolge klagt der Zwerg Dave Flood, der seinen Unterhalt mit diesem »Sport« verdient, in Forida gegen ein Verbotsgesetz aus dem Jahr 1989.

15 J. Habermas, Die Zukunft der menschlichen Natur. Auf dem Weg zu einer liberalen Eugenik, 2001, S. $70 \mathrm{f}$.

16 F.A.Z. Nr. 289 vom 12.12. 2001, S. 11. 
rem Maße ist der Begriff der »Würde des Menschen« ein solcher Grenzbegriff, der nach der einen Seite auf das Feld der Biologie und Medizin, nach der anderen auf das der Moralphilosophie hinausweist. Die dort existierenden Ungewissheiten, Zonen des Nichtwissens und Kontroversen nimmt der verfassungsrechtliche Begriff in sich auf. Sein juristischer Gebrauch dispensiert nicht von der Reflexion auf diesen Hintergrund. Man kann dies auch »diskursfreundlich « ausdrücken: Es ist gerade ein Charakteristikum unserer auf der Achtung der Menschenwürde aufgebauten Verfassungs- und Rechtsordnung, dass sie, von einem Begriffskern abgesehen, ganz unterschiedliche Auffassungen über Subjekt, Gegenstand des Schutzes und mögliche Verletzungshandlungen hinsichtlich der »Menschenwürde « zulässt. Damit respektiert die Verfassung einen Freiraum des Gewissens des Einzelnen, indem sie nicht eine Normativität vorgibt, die sie aus sich heraus nicht zu valutieren vermag. Aus seiner naturwissenschaftlichen Sicht hat Claus R. Bartram diesen Sachverhalt klar formuliert: »Wir müssen einfach erkennen, dass es zu diesen wichtigen Lebensfragen sehr unterschiedliche Auffassungen gibt, die sich auch nicht weiter annähern lassen. Dennoch kann man den anderen mit seiner Meinung doch respektieren. Da das Embryonenschutzgesetz in jedweder Fassung umstritten bleibt, wird es sicherlich auch von jeder Generation wieder neu auf den Prüfstand gestellt werden. $«{ }^{18}$

Und aus der Sicht des Verfassungsrechts liest sich, aus kompetentem Munde formuliert, das erkenntnistheoretische Dilemma, das mit den Grenzbegriffen Lebensschutz und Würdeschutz aufbricht, wie folgt: »Ich wollte vor allem deutlich machen, dass es bei der Frage nach dem Beginn des Würdeschutzes menschlichen Lebens um eine wertende Entscheidung, nicht aber um eine Frage der schlichten Rechtserkenntnis geht. Die Rechtswissenschaft ist nicht kompetent, die Frage zu beantworten, wann menschliches Leben beginnt. Das war auch nicht ... Gegenstand der Entscheidungen des BVerfG zum Schwangerschaftskonflikt. Die Naturwissenschaft ist auf Grund ihrer Erkenntnisse nicht in der Lage, die Frage zu beantworten, ab wann menschliches Leben unter den Schutz der Verfassung gestellt werden sollte. « ${ }^{19}$

Für die verfassungsrechtliche Beurteilung bedeutet diese wohlbegründete theoretische Zurückhaltung, dass die Verfassung selbst den Übergang von den auf verschiedenen disziplinären Ebenen zu führenden theoretischen Diskursen zu einem praktischen Diskurs fordert, der als rechtspolitischer Diskurs normativen Charakter annimmt. Die Maßstabsfunktion der Verfassung selbst enthält die Aufforderung, die Auseinandersetzung auf der rechtspolitischen Ebene weiterzuführen (was derzeit geschieht). ${ }^{20}$ Dies unterscheidet die freiheitliche Verfassung einer pluralistischen Gesellschaft von den heiligen, geoffenbarten Texten einer Offenbarungsreligion. Während diese ihre handlungsleitende Kraft einer authentischen, autoritativen Interpretation und Verkündi-

17 Instruktiv die Grundsatzentscheidung BVerfGE 67, 213 ( 1984, Anachronistischer Zug).

18 C.R. Bartram, Warum auf den Ethikrat warten?, F. A. Z., Nr. 148, 29. 6. 2001, S.46.

19 So die ehemalige Präsidentin des Bundesverfassungsgerichts Jutta Limbach. Vgl. Mensch ohne Makel, in F. A. Z. Nr. 47 vom 25. 2. 2002, S. 51.

20 In diesem Sinne, nämlich den Verfassungswertungspluralismus als Herausforderung an die Klugheit des Gesetzgebers zu begreifen, verstehe ich auch die Ausführungen von I. Gutiérrez Gutiérrez, El debate alemán sobre la investigación con células-madre de origen embrio- 
gung verdanken, bewährt sich jene, die Verfassung, in der Eröffnung und Absicherung eines Raumes politischer Auseinandersetzung zwischen autonomen Individuen. Oder, um es in einer zugegebenermaßen unzulänglichen, weil noch stärker verräumlichenden Sprache zu sagen: Der von der Verfassung eröffnete Gestaltungsspielraum des Gesetzgebers kann nicht mehr einfach als das auszufüllende Feld innerhalb eines ziemlich fest vorgegebenen Rahmens beschrieben werden, wie das sonst meistens genügt, sondern der Rahmen selbst bedarf erst noch der Vervollständigung durch den Gesetzgeber.

In diesem Zusammenhang sei eine Anmerkung zur öffentlichen »Streitkultur « in Deutschland erlaubt: Wenn bei einer solchen Diskussion über fundamentale Begriffe der Verfassung wie »Würde des Menschen« oder »Leben« die Auffassungen stark divergieren oder auch zusammenprallen, so darf dies kein Austausch von »Ohrfeigen « werden und sollte in den Medien auch nicht als solcher bezeichnet werden. Konkret: Wenn die Enquete-Kommission »Recht und Ethik der modernen Medizin« des Deutschen Bundestages sich mehrheitlich für ein Verbot des Importes embryonaler Stammzellen (ES) ausspricht und sich damit gegen den Standpunkt der Deutschen Forschungsgemeinschaft wendet, dann entspricht es nicht der »Würde « aller Beteiligten und nicht der Bedeutung des Streites, hier von einer »laut schallenden Ohrfeige« zu sprechen. ${ }^{21}$

\section{III.}

Die aktuelle bioethische Diskussion kreist nicht (mehr) um Fragen der Zulässigkeit der Schwangerschaftsunterbrechung; zu diesen hat das Bundesverfassungsgericht 1993 einen modus vivendi skizziert, den der Gesetzgeber in Paragraphen gegossen und damit einen, wie gesagt, freilich prekären Rechtsfrieden herbeigeführt hat. Heute sind es im wesentlichen drei ganz unterschiedliche Verfahren, denen jedoch gemeinsam ist, dass es ihnen um den Embryo in vitro geht, mithin um menschliches Leben in der Phase vor der Nidation. Die Rede ist

a) von der Präimplantationsdiagnostik (PID),

b) von der Gewinnung von und Forschung mit Embryonalen Stammzellen ( ES-Zellen) und

c) vom sogenannten Therapeutischen Klonen.

Alle drei Verfahren sind in Deutschland nach dem derzeit geltenden Gesetz zum Schutz von Embryonen vom 13. Dezember 1990 verboten und mit Strafe bedroht; z.B. aber in Großbritannien und in den Vereinigten Staaten zugelassen. Die Strafbarkeit beruht in vielen Fällen darauf, dass das Gesetz die künstliche Befruchtung einer Eizelle, die In-vitro-Fertilisation (IVF), nur zu dem Zweck erlaubt, eine Schwangerschaft der Eispenderin herbeizuführen, ferner darauf, dass das Gesetz sowohl die befruchtete, entwicklungsfähige Eizelle vom Augenblick der Kernverschmelzung an

nario, in: TRC No. 10-11 (2002/2003), 533 ff., 546. Der Autor gibt einen fundierten Überblick über die deutsche PID- und Stammzellen-Diskussion.

21 C. Schwägerl, Schallende Ohrfeige, F.A.Z. Nr. 265, vom 14. 11. 2001, S. 49. 
als Embryo und damit als Schutzgut definiert als auch jede einem Embryo entnommene totipotente Zelle, die die Teilungsfähigkeit besitzt und sich zu einem neuen Individuum zu entwickeln vermag, § 8 EmbrSchG. Die Totipotenz einer frühen Embryonalzelle oder Stammzelle, das heißt, ihre Fähigkeit, durch weitere Teilung die unterschiedlichsten Gewebearten, Nervengewebe, Muskelgewebe, Knochengewebe usw. auszubilden, macht diese Zellen für die therapeutisch orientierte biologische Forschung so außerordentlich interessant und wertvoll; man geht heute davon aus, dass die Totipotenz längstens bis zum Achtzell-Stadium des Embryos andauert, danach kommt nur noch eine »Pluripotenz « der Zelle in Betracht. ${ }^{22}$

(a) Bei dem Verfahren der Präimplantationsdiagnostik werden in vitro hergestellte Embryonen - ich verwende zur Verdeutlichung des Unterschieds zur »natürlichen « Zeugung und Fortpflanzung absichtlich die Sprache der Technik - Embryonen, die zur Herbeiführung einer Schwangerschaft eingepflanzt werden sollen, daraufhin untersucht, ob ihr Genom Anomalien aufweist, die die Wahrscheinlichkeit späterer Erbkrankheiten befürchten lassen. Ergibt sich ein solcher Befund, so bleibt es der Frau überlassen, ob sie sich für oder gegen eine Implantation des untersuchten Embryos entscheidet. Weltweit sind bisher mehr als 400 Kinder nach einer PID geboren worden. ${ }^{23}$ Die Frau kann sich straflos gegen eine Schwangerschaft überhaupt oder jedenfalls zu diesem Zeitpunkt entscheiden. Wurden ihr in dem betreffenden Zyklus drei Eizellen entommen und, was das Gesetz zuläßt, in vitro befruchtet, so sind die entstandenen Embryonen nach einer negativen Entscheidung der Frau gewissermaßen »verwaist«. Ähnlich verhält es sich, wenn aus anderen Gründen mehr Eizellen befruchtet wurden als übertragen werden können. Sie werden kryokonserviert und, falls sie nicht später doch noch auf die Frau übertragen werden, nach einiger Zeit »entsorgt«. Embryonen mit schweren genetischen Defekten können auch gleich nach der Untersuchung verworfen werden. In Deutschland existieren derzeit noch ca. 70 kryokonservierte Embryonen, die von 29 Paaren stammen. ${ }^{24}$ Weit höher ist allerdings die Zahl der im Vorkernstadium, also vor der Kernverschmelzung, konservierten Eizellen. Sie wird in Deutschland mit 32.123 angegeben. ${ }^{25}$ Andere sprechen sogar von 100.000 Konserven. ${ }^{26}$ Diese große quantitative Differenz zwischen Embryonen und »Prä-Embryonen « wird nach einem Blick in das Embryonenschutzgesetz vom 13. Dezember 1990

22 Nur noch »pluripotente« Zellen fallen nicht unter den Schutz des EmbrSchG ( $\arg$. § 8 I ).

23 Zahlenangabe nach Sigrid Graumann, Zur Problematik der Präimplantationsdiagnostik, in: Aus Politik und Zeitgeschichte, 29. Juni 2001, B 27/ 2001, 17. Die Stellungnahme des Nationalen Ethikrates, Genetische Diagnostik vor und während der Schwangerschaft, 2003, nennt eine Schätzung von weltweit 700 bis 1000 Geburten nach einer PID. Das PGD-ESHRE-Konsortium berichtet von 279 Kindern in 215 Geburten von 1994 bis Mai 2001, s. S. 57 der Stellungnahme des Ethikrates.

24 Zahlenangaben nach Frau Dr. Karin Bock, Frauenklinik der Universität Marburg, vorgetragen auf dem Bioethik-Symposium des Hessischen Landtags am 17. November 2001. Ganz andere Zahlen hört man aus Italien. Dort seien bis April 200124.276 kryokonservierte Embryonen registriert, meldet Corriere della sera am 19. 6. 2002, S. 16. Und, folgt man Maria Böhmer, F.A.Z. Nr. 192, vom 20. 8. 2002, S. 33, so »lagern ganze potentielle Kleinstädte in Kühlhäusern « in Belgien.

25 Karin Bock, s. vorige Note. 
verständlicher: Der Gesetzgeber legt die Zäsur, mit welcher der Schutz des menschlichen Lebens als Schutz des »Embryos« beginnen soll, auf den Zeitpunkt der Kernverschmelzung, also ungefähr acht Stunden nach der Befruchtung, nach dem Eindringen der Samenzelle in die Eizelle. Allerdings wird die befruchtete Eizelle nach diesem Zeitpunkt, vom Gesetz nunmehr als Embryo bezeichnet, nur in einzelnen Beziehungen, vor allem gegen missbräuchliche Verwendungen ( 2 ESchG) durch die Androhung strafrechtlicher Sanktionen geschützt; ihre schlichte Vernichtung bleibt straflos. Dies gilt auch für die durch fortgesetzte Zellteilung sich entwickelnde Blastozyste bis zum »Abschluss der Einnistung des befruchteten Eies in der Gebärmutter«, wie der Strafgesetzgeber in $\S 218$ Abs.1 StGB sagt. Dieser Zeitpunkt, die Nidation, markiert die zweite bedeutsame Zäsur, welche die Rechtsordnung in dem biologischen Kontinuum der Entwicklung menschlichen Lebens setzt. Von diesem Augenblick an bis zum Beginn der Geburt (vgl. § 217 StGB früher) rechnet die Phase der »Schwangerschaft«, deren Abbruch auch nach gegenwärtig geltendem Recht grundsätzlich rechtswidrig und strafbar ( $\$ 218$ StGB), bei Einhaltung bestimmter Voraussetzungen und Verfahren jedoch straflos, aber rechtswidrig ( $\$ 218 \mathrm{a}$ Abs. 1 StGB), und unter wieder anderen Voraussetzungen (der sogenannten Indikationslagen, § 218 Abs. 2 und 3) sogar rechtmäßig ist. Die Gesetzessprache zeichnet diese Zäsuren nach, indem sie früher - die »Frucht« oder »Leibesfrucht «, heute »das ungeborene Leben« oder das $»$ Ungeborene $«{ }^{27}$ von dem mit der Geburt »auf die Welt kommenden « oder ins »Leben tretenden« Kind unterscheidet. Übrigens sind beide Umschreibungen schief und unzulänglich: Auch der Embryo oder Fötus im Mutterleib ist schon »auf der Welt « und als »Ungeborenes « oder »nasciturus « ist er unstreitig selbst bereits »Leben«

Wesentlich problematischer, sowohl hinsichtlich der naturwissenschaftlichen Begriffsbildung als auch in der Frage des verfasssungsrechtlich gebotenen Schutzes, stellt sich die Phase zwischen der in utero oder in vitro erfolgten Befruchtung und der Nidation dar, also das im wesentlichen von dem deutschen Embryonenschutzgesetz von 1990 und von dem spanischen Gesetz Nr. 35 /1988 umspannte Stadium. In diese Phase fallen die rechtlich wie ethisch umstrittenen Tatbestände der sogenannten »embryonenverbrauchenden « Stammzellen-Forschung und des »therapeutischen Klonens «.

(b) Die Forschung mit embryonalen Stammzellen zu therapeutischen Zwecken macht sich deren Fähigkeit zur Teilung und damit Selbstvermehrung und vor allem zur Ausdifferenzierung unterschiedlicher Gewebearten zunutze. In vitro können auf diese Weise die Vorläuferzellen für Nerven, Blut oder Herzmuskelgewebe hergestellt werden. Der Embryo wird dabei möglicherweise »verbraucht«, er geht zugrunde. Transplantiert man das aus den Stammzellen entstandene spezifische Gewebe in erkrankte Organe, so sollen diese, so hofft man, durch die eingepflanzten gesunden Zellen wieder voll funktionsfähig werden. Am Institut für Neurophysiologie der Universität Köln ist es vor anderthalb Jahren gelungen, durch Herzinfarkte geschädigte Mäuseherzen durch Behandlung mit aus embryonalen Stammzellen gewonnenen Vor-

26 Reinhard Merkel, Verfügen über Leben und Tod, Vortrag bei den »Frankfurter Tagen der Rechtspolitik « am 6. 12. 2001, Aula der Universität Frankfurt.

27 Lies: § 219 Abs.1 StGB. 
läuferzellen, sogenannten »schlagenden Inseln«, wieder zu normalem Herzschlag zu bringen. ${ }^{28}$ Was bei Mäusen vielversprechend gelungen ist, muss nicht auch beim Menschen Erfolg haben; immerhin eröffneten diese und ähnliche Versuche eine konkrete Forschungsperspektive. Jüngste Berichte stimmen hier jedoch eher skeptisch. Zu den zahlreichen noch ungelösten Einzelproblemen gehört die Vermeidung von Abstoßungsreaktionen, die beim Zusammenwachsen von Zellen mit unterschiedlichen Erbgutinformationen auftreten.

(c) Eben dieser Problematik hofft man durch ein Verfahren zu entgehen, das man als therapeutisches Klonen durch Zellkerntransfer bezeichnet. Der Grundgedanke ist, dass dem kranken Organ des Patienten nur solche heilenden Zellen zugeführt werden, deren genetische Struktur mit der des Empfängers identisch ist. Seine Verwirklichung wird durch die »wunderbar« zu nennende Fähigkeit bestimmter Zellen zur »Reprogrammierung « ermöglicht. Um dies zu erläutern, sei hier das Verfahren skizziert: Aus der Eizelle wird der die Erbinformationen tragende Zellkern entfernt. Statt dessen wird der aus einer somatischen Zelle des Patienten/der Patientin (also aus einer sogenannten adulten Zelle) isolierte Zellkern in die Eizelle eingebracht, in welcher der adulte Kern nach entsprechender Aktivierung sich zu teilen beginnt und in den frühembryonalen Zustand zurückverwandelt, »reprogrammiert«, wird. Wie bei einem normalen Befruchtungsvorgang entsteht in den folgenden Tagen eine Blastozyste, aus deren innerer Zellmasse sich selbst vermehrende Stammzellen gewonnen werden können, während der Embryo zerstört wird. Die gewebespezifisch ausdifferenzierten Stammzellen können dann auf den/die Patienten(in) transplantiert werden und zur Organneubildung aus den »körpereigenen«, genetisch identischen Stammzellen führen. Hier wird also zu Heilzwecken, therapeutisch, geklont. Würde man hingegen die Blastozyste einem Uterus einpflanzen und den Fötus austragen lassen, entstünde eventuell ein geklonter Mensch, das heißt, gemäß der Definition des Zusatzprotokolls des Europarats zur Biomedizin-Konvention über das Verbot des Klonens, »ein menschliches Lebewesen, das mit einem anderen menschlichen Lebewesen dasselbe Kerngenom gemeinsam hat « (Art. 1 Abs.2) ${ }^{29}$ Hier spricht man von »reproduktivem Klonen«. Eine praktische Schwierigkeit für Verbreitung des therapeutischen Klonens - wie übrigens der Stammzellenforschung überhaupt - ist die Knappheit der Eizellen. 4-5000 Dollar, heißt es, erhält eine Frau in den USA, die sich als Eizellenspenderin zur Verfügung stellt und sich der unangenehmen hormonellen Stimulation unterzieht. Junge, attraktive Frauen mit Hochschuldiplom seien besonders begehrt, für die Vermittlung seien bis zu 30000 Dollar »Gebühr « üblich ${ }^{30}$. Im übrigen sind jüngste amerikanische Versuche des reproduktiven Klonens mit Rhesusaffen so negativ verlaufen, dass die Mög-

28 S. Pieplow, Stammzell-Ersatz nach Herzinfarkt, F.A.Z. Nr. 283, 5.12. 2001, s.N 1.

29 Zusatzprotokoll des Europarats zur Konvention über das Verbot des Klonens von menschlichen Lebewesen, Art. 1 Abs. 2. Vgl. auch die Menschenrechtskonvention zur Biomedizin des Europarats vom 4. 4. 1997, die zwar in Kraft getreten, jedoch von der Bundesrepublik nicht ratifiziert worden ist.

30 Vgl. Martina Keller, Frische Eizellen, zu Tausenden gesucht. Frankfurter Allgemeine Sonntagszeitung vom 9. 12. 2001, S. 65. 
lichkeit einer Übertragung des Verfahrens der Herstellung von embryonalen Stammzellen beim Menschen auch nur zu therapeutischen Zwecken nunmehr ernsthaft bezweifelt wird. ${ }^{31}$

\section{$I V$.}

Die rechtliche Beurteilung der skizzierten Verfahren durch die europäischen Gesetzgeber fällt unterschiedlich aus. Das reproduktive Klonen wird, soweit ersichtlich, ${ }^{32}$ überall abgelehnt und vielfach ausdrücklich verboten. Hier ist Art. 1 des zitierten Zusatzprotokolls zur Biomedizin-Konvention des Europarates vom 4.4.1997 ebenso zu nennen wie schon $\S 6$ des deutschen Embryonenschutzgesetzes von 1990 und Art. 3 Abs. 2 der Charta der Grundrechte der Europäischen Union. Die Erläuterung zu dieser Vorschrift betont, dass die Charta andere Formen des Klonens, also etwa das therapeutische Klonen, weder gestattet noch verbietet. Die Entscheidung hierüber bleibt den Rechtsordnungen der Mitgliedstaaten überlassen. Die Biomedizin-Konvention von 1997 lässt die Forschung an Embryonen in vitro, also auch an Stammzellen, nach Maßgabe der staatlichen Rechtsordnungen $\mathrm{zu}$, fordert aber einen »angemessenen Schutz des Embryos«. Hingegen ist die Herstellung menschlicher Embryonen zu Forschungszwecken verboten (Art. 18). Es fehlt auch an einer klaren und ausdrücklichen Aussage der Konvention zur Präimplantationsdiagnostik; immerhin sollen prädiktive genetische Tests bei einer »Person« für »Gesundheitszwecke oder für gesundheitsbezogene wissenschaftliche Forschung « unter bestimmten Voraussetzungen zulässig sein. (Art. 12). Unklar bleiben der Personbegriff und der Bezug auf die Gesundheit. Nach dem derzeit geltenden deutschen Embryonenschutzrecht sind sowohl das therapeutische Klonen als auch die Forschung an Embryonen oder an totipotenten Stammzellen ebenso wie die Erzeugung von Embryonen in vitro zu Forschungszwecken als auch die Präimplantationsdiagnostik (PID) verboten und strafbar.

\section{$V$.}

Nach dieser bisher nur deskriptiven Betrachtung der biologischen Sachverhalte und dem kurzen Blick auf die in vielerlei Hinsicht unbefriedigende Gesetzeslage gilt es nunmehr, das Problem des Embryonenschutzes und insbesondere des Schutzes der in vitro existierenden Embryonen vor einer Nidation, zu der die meisten von ihnen nie gelangen, normativ zu betrachten. Diese Betrachtung kann sich primär an philosophisch-ethischen oder primär an verfassungsrechtlichen Maßstäben orientieren, soweit solche überhaupt nachvollziehbar und verallgemeinerbar existieren. Denkbar ist auch,

31 Vgl. Joachim Müller-Jung, Das Klonen am Scheideweg, F.A.Z., Nr. 86, vom 11. 4. 03, S. 38.

32 Vgl. die informative Darstellung von Hans-Georg Koch, Fortpflanzungsmedizin im europäischen Rechtsvergleich, in: Aus Politik und Zeitgeschichte, Beilage zu: Das Parlament, B 27/ 2001, S. 44 ff. Zur gesamten Problematik aus schweizerischer Sicht; Rainer J. Schweizer, Verfassungs- und völkerrechtliche Vorgaben für den Umgang mit Embryonen, Föten sowie Zellen und Geweben. Zürich, Basel, Genf 2002. 
dass die verfassungsrechtliche Diskussion, wie schon einleitend bemerkt, theoretisch nur vor einem offenen philosophischen Horizont und praktisch deshalb nur in einem komplementären gesetzgebungspolitischen Diskurs geführt werden kann. Als einer, der das verfassungsjuristische Handwerk gelernt hat, beginne ich mit der Kritik der Rechtsprechung des Bundesverfassungsgerichts, welche die Aussagen des Grundgesetzes autoritativ konkretisiert hat. Danach hat die staatliche Schutzpflicht für das »ungeborene« menschliche Leben ihren Grund im Menschenwürde-Satz des Art. 1 Abs. 1 GG, welcher alle staatliche Gewalt zur Achtung und zum Schutz der Würde des Menschen verpflichtet; der Gegenstand und das Maß der Schutzpflicht werden hingegen, so das Gericht, durch Art. 2 Abs. 2: »Jeder hat das Recht auf Leben und körperliche Unversehrtheit « und: in dieses Recht »darf nur auf Grund eines Gesetzes eingegriffen werden«, näher bestimmt. Während also das Lebensrecht unter Gesetzesvorbehalt steht, das heißt, dass es der Gesetzgeber mit anderen hochrangigen Verfassungsrechtsgütern und Grundrechten abwägen und zu einem verhältnismäßigen Ausgleich bringen darf, wird aus der »Unantastbarkeit« der Menschenwürde (Art. 1 Abs. 1 Satz 1 GG) ihr Anspruch auf absoluten Schutz hergeleitet. Die »Menschenwürde als Wurzel aller Grundrechte ist mit keinem Einzelgrundrecht abwägungsfähig«, sagt das Bundesverfassungsgericht ${ }^{33}$. In einer grundlegenden früheren Entscheidung aus dem Jahr 1971, die den Konflikt zwischen postmortalem Persönlichkeitsschutz als Ausfluss der Unverletzlichkeit der Menschenwürde mit der Kunstfreiheitsgarantie betraf, ist allerdings, - mit der späteren Judikatur nur schwer vereinbar -, davon die Rede, die Entscheidung über die Zurückdrängung der Kunstfreiheitsgarantie wegen eines schweren Eingriffs in den schutzwürdigen Persönlichkeitsbereich könne »nur unter Abwägung aller Umstände des Einzelfalles getroffen werden «. ${ }^{34}$ Ohne auf Einzelheiten und Unstimmigkeiten der »Abwägungsdogmatik « hier näher einzugehen, bleibt festzuhalten, dass eine überzeugende Bestimmung des Verhältnisses von Lebensschutz zu Würdeschutz die Schlüsselfrage für alle weiteren verfassungsrechtlichen Überlegungen in unserem Problembereich bildet.

Das Bundesverfassungsgericht lässt das Lebensrecht des Ungeborenen als sein elementares und unveräußerliches Recht von der Würde des Menschen »ausgehen«, es gelte »unabhängig von bestimmten religiösen oder philosophischen Überzeugungen, über die der Rechtsordnung eines religiös-weltanschaulich neutralen Staates kein Urteil zusteht. ${ }^{35}$ Die, wie zu Recht kritisiert wurde ${ }^{36}$, ohne jede Begründung behauptete enge Koppelung von Lebensrecht und Menschenwürdeschutz, für die das Gericht außerdem ebenfalls ohne ein Wort der Begründung einen absoluten, in diesem Sinne objektiven, also standpunktunabhängigen, ideologie-, religions- und philosophieunabhängigen Geltungsanspruch postuliert, konzentriert sich in der Formel:»Wo menschliches Leben existiert, kommt ihm Menschenwürde $\mathrm{zu} \ll$. Soll dies eine positivrechtliche

33 BVerfGE 93, 266, 293; ähnlich schon E 75, 369, 380 für das »allgemeine Persönlichkeitsrecht«, soweit es »unmittelbarer Ausfluss der Menschenwürde ist«, 1987.

34 BVerfGE 30, 173, 195 (»Mephisto «).

35 BVerfGE 88, 203, 252, auch zum folgenden Zitat des Urteils.

36 Kyrill-A. Schwarz, »Therapeutisches Klonen« - ein Angriff auf Lebensrecht und Menschenwürde des Embryos? KritV 2/ 2001, 182 ff., 200. 
Feststellung oder eine naturrechtliche Begründung des Rechtes auf Leben oder eine metaphysikfreie, nämlich philosophieunabhängige Aussage über das Wesen des Menschen sein? Die erstere würde schon an dem Widerspruch zwischen der Absolutheit des Menschenwürdesatzes und der Relativität des Lebensrechtes scheitern. ${ }^{37}$ Die zweite führt, wie man sogleich sehen wird, zu biologistischen, »naturalistischen « Fehlschlüssen und die dritte kann widerspruchsfrei nicht einmal gedacht werden. »Würde des Menschen« ist kein naturwissenschaftlich feststellbarer Sachverhalt, sondern ein normativer, geschichts- und kulturbezogener Zuschreibungsbegriff, der ohne einen religiösen oder weltanschaulich-philosophischen Standpunkt gar nicht gebildet werden kann. Die , biologistische Falle' schnappt denn auch sogleich zu, wenn man die Interpretation der Koppelungsthese durch den ehemaligen Präsidenten und Vorsitzenden des ersten Senats des Bundesverfassungsgerichts und somit einen Mitautor jener Formel aus dem ersten Urteil 1975 zum Abtreibungsrecht ${ }^{38}$, nämlich durch Ernst Benda, heranzieht. Er sagt: »Wo man Leben zerstört, braucht man über die Menschenwürde des hiervon Betroffenen nicht mehr zu reden. Mit dem Leben ist eben alles weitere auch zerstört.« Dieser Satz kann schon nach den Konsequenzen, die das Gericht selbst bezüglich der Rechtmäßigkeit der medizinischen, der kriminologischen oder der embryopathischen Indikation oder gleichzuachtender Tatbestände zieht, nicht zutreffen. In diesen Fällen muss auch das Gericht davon ausgehen, dass nicht jede Lebenszerstörung, hier des Embryos, ohne weiteres zugleich eine Verletzung, eine »Antastung « der Menschenwürde bedeuten muss. Insoweit sind die beiden Schutzbereiche des »Lebens « und der »Menschenwürde« unabhängig voneinander zu bestimmen. Schon vor zehn Jahren habe ich dies am Beispiel der sogenannten »Früheuthanasie« für Anencephale, für schwerstgeschädigte Neugeborene, die ohne Schädeldecke und Großhirn zur Welt kommen, erörtert. ${ }^{39}$

Auf der einen Seite sind Verletzungen der »Würde« denkbar - und leider nicht nur denkbar! - nämlich »Erniedrigungen«, Zerstörungen der personalen Identität, grundsätzliche Infragestellungen der »Subjektqualität«, welche die physisch-biologische Existenz des Individuums, sein »Leben« im Sinne des Art. 2 Abs. 2 GG unangetastet lassen. Die Europäische Menschenrechtskonvention erkennt dies an, indem sie neben dem Recht auf Leben in Art. 2 in Art. 3 einen eigenständigen Schutzbereich garantiert: No one shall be subjected to torture or to inhuman or degrading treatment or punishment. Auf der anderen Seite dürfte, hätte Ernst Benda recht, die Rechtsordnung keine Beschränkungen oder gar Vernichtungen des Lebens zulassen, denn dann würde mit diesen zugleich auch die unantastbare Würde eben doch angetastet. Nicht nur die Indikationen beim Schwangerschaftsabbruch wären dann unzulässig, sondern ebenso die Rechtspflicht des Soldaten, notfalls sein Leben aufzuopfern, und freilich auch der

37 Übrigens spricht das GG, das hier positivrechtlich allein maßgeblich ist, mit Bedacht von der Würde des Menschen und nicht von der Würde menschlichen Lebens. Dass beides nicht identisch ist, ergibt sich aus dem im Text Folgenden.

38 S. BVerfGE 39, 1, 41, Urteil vom 25. 2. 1975.

39 E. Denninger, Rechtsethische Anmerkungen zum Schwangerschaftsabbruch und zur sogenannten »Früheuthanasie«, KJ 1992, 282 ff., 287 f. S. auch meinen Beitrag in S. Rodotà (a cura di), Questioni di bioetica, Roma/Bari 1993, S. 342 ff. 
»finale Rettungsschuss « des Polizeibeamten, der den Geiselnehmer tötet. Auf die geschichtlich-kulturelle Relativität der Beziehung zwischen Lebensschutz und Achtung der Würde sei hier nur am Rande mit den Beispielen »Soldatentod« und »Todesstrafe« aufmerksam gemacht. Der Soldat starb, jedenfalls früher, den »Heldentod « auf dem »Felde der Ehre«; seine Würde blieb vollkommen gewahrt. Und die Strafe, auch die Todesstrafe, die bei Mord zwingend notwendig eintreten müsse, ist für Hegel (1820) nicht nur an sich gerechte Vergeltung, sondern dass sie als in der Handlung des Verbrechers liegend »als sein eigenes Recht enthaltend angesehen wird, darin wird der Verbrecher als Vernünftiges geehrt. ${ }^{40}$ Heute würde in Deutschland die Wiedereinführung der Todesstrafe nach wohl überwiegender, aber umstrittener Auffassung als unzulässiger Verstoß gegen den Menschenwürdesatz angesehen werden. ${ }^{41}$

Was ist nun mit der hier vorgeschlagenen Entkoppelung der Bestimmung der beiden Schutzbereiche voneinander für die Beantwortung der uns bedrängenden Fragen nach der Zulässigkeit einer Embryonen oder Stammzellen »verbrauchenden« Forschung, der Herstellung oder des Imports von Embryonen, nach der Zulässigkeit des »therapeutischen Klonens« oder der Präimplantationsdiagnostik gewonnen ?

Zunächst nicht mehr, aber auch nicht weniger als die Erkenntnis, dass ein Eingriff in das Leben des Embryos in vitro - denn wir beschränken unsere Betrachtung hier auf die Phase vor der Nidation - nicht schon per se eine Würdeverletzung bedeutet und damit verfassungsrechtlich verboten ist. Das Leben der Zygote und des frühen Embryos - sofern man hier überhaupt schon von menschlichem Leben sprechen will genießt nur relativen verfassungsrechtlichen Schutz; es kann gegen andere Rechte und Güter abgewogen werden. Die gegenwärtige Embryonenschutz-Debatte zeigt, dass dabei auch die Frage nach dem Beginn des Lebens längst noch nicht erledigt ist. ${ }^{42}$ Es gibt zahlreiche und gewichtige biomedizinische und biologische (Markl, Nüßlein-Volhard, Bartram) ${ }^{43}$, rechtswissenschaftliche (Anderheiden, Lege, Mahrenholz, Herdegen, Monika Frommel, Wolfrum (?)) und sogar theologisch-philosophische (R. Schröder) ${ }^{44}$ Stimmen, welche den Beginn individuellen Lebens und seines Schutzes in vollem Umfang nicht einfach schon mit der Kernverschmelzung der Gameten annehmen wollen, die vielmehr in dem Vorgang der Nidation (bzw. der Implantation) eine entscheidende Zäsur sehen. ».. erst mit der Einnistung in den Uterus der Mutter hat der Embryo das volle Entwicklungsprogramm. Erst während dieser erstaunlichen und wundersamen Symbiose wird das Programm ausgeführt. Gene sind nicht alles, was

40 G. W. F. Hegel, Grundlinien der Philosophie des Rechts, Werke Band 7, 1970, § 100, § 101, Zusatz. Hervorhebungen i.O.

41 Zum Streitstand einerseits Kunig in v.Münch/Kunig, GG, 1996, Art. 102 Rdn. 18; Podlech in AK-GG, 3. Aufl. 2001, Art. 102 Rdn. 46 ff.; andererseits Dreier in Dreier GG, III, 2000, Art. 102 Rdn. 54 ff. jeweils m.Nachw.

42 Insofern hat meine 1992 , a.a.O. S. 285, geäußerte Hoffnung getrogen.

$43 \mathrm{Zu}$ Markl und Nüsslein-Volhard s.u., zu Bartram s. N. 18.

44 M. Anderheiden, »Leben« im Grundgesetz, KritV 2001, 353 ff.; zu Herdegen, Lege, Mahrenholz vgl. Expertengespräch im BMJ am 28. 6. 2001, genehmigtes Protokoll, S. 66, bzw. 47, 50; zu Herdegen s. auch JZ 2001, 773 ff.; M. Frommel, KJ 2000, 341 ff., 349; zu Wolfrum und Schröder s.u. 
der Mensch zur Menschwerdung braucht«, schreibt die Nobelpreisträgerin Christiane Nüsslein-Volhard ${ }^{45}$, und der inzwischen ausgeschiedene Präsident der Max-PlanckGesellschaft, Hubert Markl, kommt in seiner Erwiderung auf die Grundsatzrede des Bundespräsidenten vom 18. Mai 2001 in nicht weniger grundsätzlichen Überlegungen zu dem Ergebnis: »Die eigentliche, biologische Entscheidung ' zur Menschwerdung fällt daher tatsächlich mit der Einnistung des Keimes im Uterus, nicht schon mit der Befruchtung. ${ }^{46}$ Mit der Betonung der besonderen Bedeutung des Mutterleibes für die Menschwerdung befinden sich die genannten Naturforscher in Einklang mit Repräsentanten nicht nur der jüdischen sondern auch der evangelischen Theologie. Richard Schröder etwa antwortet auf die Frage: Wie wird ein Mensch ein Mensch? : »Nicht ohne die Befruchtung einer Eizelle, aber auch nicht ohne seine Mutter. $\ll^{47}$ Und nach Auffassung des Rechtswissenschaftlers Rüdiger Wolfrum verdrängt »die argumentative Gleichsetzung von Embryonen außerhalb und innerhalb des Mutterleibes « »die Bedeutung der Mutter für die Entwicklung zum Menschen und überbetont die Bedeutung der genetischen Disposition. Sie wird dem Phänomen der Menschwerdung und der Individualität nicht gerecht. ${ }^{48}$ Diese diskursorientierte Redeweise deutet die Richtung an, in der wir weiterdenken sollten. Das Ziel ist ja nicht die ohnehin unmögliche Erarbeitung eines unanfechtbar-unangefochtenen theoretischen Begriffs des »Lebens « - s. meine Einleitungsbemerkung zu »Identitätskonflikten« - sondern das Ziel muss eine rechtspraktische Konsensfindung über die Möglichkeiten und die Grenzen des forschenden und therapeutischen Umgangs mit Embryonen und Stammzellen in vitro sein, Findung eines Konsenses der den auch grundrechtlich geschützten Belangen der Wissenschaftsfreiheit und der Therapiefreiheit ebenso »gerecht « zu werden versucht wie ethischen Vorstellungen und dem - unabhängig von der Frage nach der subjektivrechtlichen Grundrechtsträgerschaft - objektivrechtlich zu gewährleistenden Lebensschutz. Dabei ist zu berücksichtigen, dass es eben in dem biologischen Werdensprozess nicht nur, wie behauptet wird, ${ }^{49}$ einen »entscheidenden qualitativen Sprung « gibt, nämlich den der Befruchtung, sondern mindestens drei Zäsuren: Kernverschmelzung, Nidation und Geburt, an welche die Rechtsordnung differenzierte Rechtsfolgen anknüpfen kann.

Entsprechendes gilt vom »Menschenwürde«-Argument. Juristen haben schon immer, und auch in unserem Zusammenhang, vor dem inflationären Gebrauch des Begriffs gewarnt. ${ }^{50}$ Richard Schröder ${ }^{51}$ meint, wer schon das mikroskopisch kleine

45 C. Nüsslein-Volhard, Wann ist ein Tier ein Tier, ein Mensch kein Mensch ? F.A.Z. Nr. 229 v. 2. 10. 2001, S. 55.

46 H. Markl, Von Caesar lernen heißt forschen lernen, F.A.Z. Nr. 144 vom 25. 6. 2001.

47 R. Schröder, Was dürfen, was sollen wir tun ? F.A.Z. Nr. 167 vom 21. 7. 2001.

48 R. Wolfrum, Forschung an humanen Stammzellen: ethische und juristische Grenzen, in: Aus Politik und Zeitgeschichte, Beilage zu: Das Parlament, B 27 / 2001, S. 3, 4.; anders jedoch derselbe, Der Embryo und sein Rechtsstatus, forschung, Magazin der DFG, 2-3/ 2001, S.2/3, hier stellt W. auf den Zeitpunkt der Befruchtung ab.

49 z.B. W. Höfling, Zygote - Mensch - Person, F.A.Z. Nr. 157 vom 10. 7. 2001, S.8.

50 Zuletzt H.-G. Koch, a.a.O. (N.31), S. 46.

51 R. Schröder, Interview in DER SPIEGEL Nr. 50/2001 vom 10. 12. 2001, S. 228. 
Gebilde der befruchteten Eizelle als »Mitmensch« an- und ihm »Menschenwürde « zuspreche, solle aufpassen, dass er diesen Begriff nicht beschädige. Dieser Autor und viele andere weisen auf die Schwierigkeit hin, den Würdebegriff mit der Tatsache zu vereinbaren, dass schon bei der natürlichen Fortpflanzung ca. 70\% der Zygoten und Embryonen »verloren gehen«, umkommen, und dass bei der In-vitro-Fertilisation die sogenannte »Baby-take-home-Rate « auf höchstens $15 \%$ geschätzt wird. ${ }^{52}$ Dies mag hier auf sich beruhen, jedenfalls tut der Jurist, der einen Begriff benutzt, »auf dem zweieinhalbtausend Jahre Philosophiegeschichte lasten $«,{ }^{53}$ gut daran, den Rat der Philosophen einzuholen. Ich widerstehe hier der Versuchung, die Mensch-, Person- und Gottwerdungs-Theorie des späten Max Scheler $(1927 / 8)^{54}$, des zu Unrecht Halbvergessenen, heranzuziehen. Vielmehr weise ich auf den Zusammenhang von Personsein und Menschenwürde hin, wie ihn Jürgen Habermas in seiner jüngsten Studie über »Die Zukunft der menschlichen Natur« unter der Fragestellung »Menschenwürde vs. Würde des menschlichen Lebens « entwickelt hat. ${ }^{55}$ Dass die Würde des Menschen an sein Personsein gebunden ist, war übrigens dem Bundesverfassungsgericht schon vor dreißig Jahren wohl bekannt, nur ist ihm dieses Bewusstsein durch die biologistische Fundierung des vorgeburtlichen Lebensschutzes in den beiden Urteilen zum Schwangerschaftsabbruch 1975 und 1993 etwas abhanden gekommen. ${ }^{56}$ In dem »MephistoBeschluss « aus 1971, in dem es nicht um den pränatalen, sondern um den postmortalen Würdeschutz ging, heißt es an zentraler Stelle, es sei mit der Unverletzlichkeit der Menschenwürde unvereinbar, wenn »der Mensch, dem Würde kraft seines Personseins zukommt, in diesem allgemeinen Achtungsanspruch auch nach seinem Tode herabgewürdigt oder erniedrigt werden dürfte. $\aleph^{57}$ Personsein ist aber nicht identisch mit »menschlichem Leben«. Habermas sieht den entscheidenden Vorgang der Personwerdung in der Geburt, hier geschehe der »gesellschaftlich individuierende Akt der Aufnahme in den öffentlichen Interaktionszusammenhang einer intersubjektiv geteilten Lebenswelt. Erst im Augenblick der Lösung aus der Symbiose mit der Mutter tritt das Kind in eine Welt von Personen ein, die ihm begegnen, die es anreden und mit ihm sprechen können. Keineswegs ist das genetisch individuierte Wesen im Mutterleib, als Exemplar einer Fortpflanzungsgemeinschaft, »immer schon« Person. Erst in der Öffentlichkeit einer Sprachgemeinschaft bildet sich das Naturwesen zugleich zum Individuum und zur vernunftbegabten Person. ${ }^{58}$ Und Habermas weist auf Hannah Arendt hin, welche dieses personkonstitutive Interaktionsverhältnis in den lateinischen Begriffen für »leben« und »sterben« wiederfindet, nämlich: inter homines esse und desinere inter homines esse, »unter Menschen weilen« bzw. »aufhören unter Men-

52 Zahlen nach Karin Bock, a.a.O. ( N. 23).

53 Pieroth/ Schlink, Grundrechte, Staatsrecht II, 15. Aufl. 1999, Rdn. 353.

54 Vgl. Max Scheler, Die Stellung des Menschen im Kosmos, 1927, 1947, 1949, S. 87, 90; zu Schelers Personlehre s. E. Denninger, Rechtsperson und Solidarität, 1967.

55 J. Habermas, Die Zukunft der menschlichen Natur. Auf dem Weg zu einer liberalen Eugenik?, 2001, S. $56 \mathrm{ff}$.

56 BVerfGE 39, 1, 41 und 88, 203, 252.

57 BVerfGE 30, 173, 194.

58 Habermas, a.a.O. (N. 54), S. 64 f. 
schen zu weilen $« .{ }^{59}$ Die alten Römer, dieses zutiefst politische Volk (H. Arendt), hatten das Gespür dafür, dass Menschsein die Mitgliedschaft in einer Gemeinschaft wechselseitiger Anerkennung bedeutet. Und dass, mit den Worten Habermas', Menschenwürde nicht eine Eigenschaft ist, »die man von Natur aus ,besitzen“ kann wie Intelligenz oder blaue Augen; sie markiert vielmehr diejenige »Unantastbarkeit«, die allein in den interpersonalen Beziehungen reziproker Anerkennung, im egalitären Umgang von Personen miteinander eine Bedeutung haben kann. ${ }^{60}$

$V I$.

Hieraus folgt nun aber keineswegs, dass das vorgeburtliche, vorpersonale Leben, sei es intrauterin als Fötus oder extrakorporal als Zygote oder Embryo in vitro, im Hinblick auf den möglicherweise daraus entstehenden Menschen und seine Würde völlig schutzlos gestellt wäre. Ebenso wie der objektivrechtliche Schutzgehalt des Grundrechts auf Leben dem Gesetzgeber eine differenzierte Schutzpflicht zugunsten des menschlichen bios auferlegt, gleichgültig, ob man den »jeder« im Sinne des Art. 2 Abs. 2 GG mit der Kernverschmelzung oder mit der Nidation beginnen läßt, und ebenso wie die Würde einer verstorbenen Person über ihren Tod hinaus fortwirken kann und z.B. einen würdegemäßen Umgang mit der Leiche fordert, so gebietet der Menschenwürdesatz des Art. 1 Abs. 1 GG, dass die Rechtsordnung einen würdegemäßen Umgang auch mit den frühesten Formen neu sich bildenden Lebens gewährleistet. Ob man hier von »Vorwirkung « und einer »Vorstufe« des Menschenwürdeschutzes oder von »potentieller Menschenwürde« spricht, ist nur von rhetorischer Bedeutung. Wichtiger ist es, Kriterien dafür zu entwickeln, welche Verhaltensweisen in diesen Stadien als Würdeverletzungen anzusehen sind und welche nicht. Dass z.B. die Folter bei dem geborenen Menschen eine solche Verletzung darstellt, ist (hoffentlich! ${ }^{61}$ ) evident, ebenso aber auch, dass das Folterverbot in Bezug auf eine Blastozyste fünf Tage nach der Fertilisation leer läuft. Weniger dramatisch müssen (spitz)findige Strafprozessualisten sich fragen, ob ein Embryo in der Petrischale oder eine entwicklungsfähige Stammzelle als Beweismittel eines Delikts (gegen das EmbrSchG) nach §§ 94 ff. StPO »beschlagnahmt « werden darf oder nicht. »Menschen« dürfen nach bisherigem Rechtsverständnis nicht beschlagnahmt, allenfalls in Haft oder in Gewahrsam genommen werden. Leichen, Leichenteile und abgetrennte Körperteile, tote Föten können beschlagnahmt werden, aber »lebende« Embryonen? Sie sind keine »Sachen« im Sinne des Gesetzes.

Es schafft insbesondere im Hinblick auf die umstrittenen Tatbestände der Präimplantationsdiagnostik, der Stammzellenforschung und des therapeutischen Klonens

59 H. Arendt, Vita activa, 5. Aufl. 1987 der Ausgabe 1981, S. 15.

60 J. Habermas, a.a.O., S. 62.

61 Die jüngst (März/April 2003) am Fall des Frankfurter Polizeivizepräsidenten Daschner entbrannte Diskussion über die Zulässigkeit polizeilicher Folter zur Rettung eines Entführungsopfers gibt allerdings zu schlimmen Befürchtungen Anlass, was künftig den Respekt vor dem Verbot der Folter angeht. 
keine Klarheit, wenn man, wie vorgeschlagen wird, ${ }^{62}$ die Würdeverletzung dann annimmt, wenn eine »Tabuverletzung « vorliegt. Das ist in einer pluralistischen Gesellschaft, die gerade permanent um ihre »Tabugrenzen« ringt, nur ein Wort, das im übrigen bei der heutigen Bewusstseinslage auch den Sachverhalt nicht richtig beschreibt. Anhaltspunkte für vielleicht konsensfähige Grenzziehungen gewinnt man, wenn man, entsprechend den Würdeverletzungen bei Personen, herabwürdigende, verächtliche, die Subjektqualität grundsätzlich in Frage stellende Behandlungsweisen ins Auge fasst. Dies ist der Fall, wenn die Embryonen oder Zellen als Ware behandelt werden, und ebenfalls, wenn sie, wie Waren für einen Markt, hergestellt und dann verbraucht werden. In diesem Sinne rechnet auch das spanische Gesetz Nr. 35 / 1988 den Handel mit »Präembryonen « oder deren Zellen, deren Import oder Export zu den besonders schweren Straftaten ( $\gg$ infracciones muy graves «) in diesem Bereich. ${ }^{63}$

In Deutschland bedeutet dies, dass eine therapiebezogene Forschung mit embryonalen Stammzellinien nur insoweit in Betracht kommt, als hierzu sogenannte »überzählige « Embryonen verwendet werden, die nicht mehr für die Herbeiführung einer Schwangerschaft verwendet werden können. Ähnliches gilt für die Beschaffung der Eizellen, die für das therapeutische Klonen notwendig werden. Hierbei ist zu beachten, dass es nur um die Heilung schwerer Krankheiten gehen darf, niemals um die ,Herstellung' neuer Lebewesen und auch nicht um die Herstellung biologischer Ersatzteile auf Lager. Auch Grenzfälle wie der, den jüngst ein britisches Obergericht zu entscheiden hatte, lassen sich unter diesen Voraussetzungen einer vernünftigen Lösung zuführen: Wenn Eltern, und sei es im Wege der assistierten Reproduktion, sich ein zweites, gesundes, Kind wünschen, mit Hilfe dessen Zellen die Krankheit des ersten Kindes möglicherweise erfolgreich bekämpft werden könnte, ohne die Gesundheit des zweiten Kindes zu beeinträchtigen, so verdient der Elternwille Berücksichtigung, es sei denn, aus den Umständen geht eindeutig hervor, dass das zweite Kind ausschließlich zum Zwecke der Heilung des ersten geboren werden sollte. Diese Betrachtungsweise steht völlig in Einklang mit der Maxime Kants, die es gebietet, das Subjekt »niemals bloß als Mittel, sondern zugleich selbst als Zweck zu gebrauchen. ${ }^{64}$

Verfahrensrechtliche und organisatorische Sicherungen zur Verhütung von Missbräuchen sind unerlässlich. Dies gilt schließlich auch für die ebenfalls restriktiv, das heißt nur zur Krankheitsvermeidung, nicht zu »eugenischen « Zwecken zuzulassende Präimplantationsdiagnostik. Auch hier muss der Gesetzgeber eingehende Regelungen treffen. Er kann einen Katalog derjenigen Anomalien und genetischen Defekte aufstellen, deren mutmaßliches Vorhandensein ein pränidatives Diagnoseverfahren rechtfertigt; er kann eine Genehmigungspflicht und ein entsprechendes Verfahren einführen und er muss auf jede vernünftige Weise dafür sorgen, dass das, was der Frau die Notwendigkeit einer embryopathisch indizierten Abtreibung ersparen soll, nicht in den

62 Höfling, a.a.O., (N. 49).

63 Art. 20 Abs.2 B. lit e) des Gesetzes über Reproducción Asistida Humana, Nr. 35 / 1988, 22.11.1988. Ebenso R. J. Schweizer, A.a.o. (N. 32), S. 9, 35 ff.

64 Kant, Kritik der praktischen Vernunft, Werkausgabe hrsg. von W. Weischedel, Band IV, Darmstadt 1956, S. 210. Zum Fall vgl. die Notiz in der F.A.Z. vom 9. 4. 2003. 
ersten Schritt einer selegierenden »eugenischen Praxis«, also der Menschenzüchtung, im Sinne des Art. 3 Abs. 2 EU-Grundrechte-Charta umschlägt. ${ }^{65}$

Wir stehen mitten in einer rasanten Entwicklung der Biomedizin. Die Menschheit, die erst vor wenigen Jahrhunderten den Verlust der kosmozentrischen Sicherheit hinnehmen musste, welche das geozentrische Weltbild bot, und die erst seit rund $150 \mathrm{Jah}-$ ren ihre durch die Evolutionstheorie relativierte Stellung in der Ordnung der Arten bestimmen muss, sieht sich heute von der neuen Unsicherheit bedroht, dass nicht einmal mehr ihre eigene Natur konstant, sondern dem beeinflussenden Zugriff durch den Menschen selbst ausgesetzt ist. Aber es wäre falsch, hier nur die Bedrohung zu sehen, nicht auch die Chancen. Niemand hat diese Situation des modernen Menschen treffender ausgedrückt als - vor mehr als zweihundert Jahren, in seiner Frankfurter Zeit Friedrich Hölderlin mit den Worten:

»Alles prüfe der Mensch, sagen die Himmlischen,/ Daß er, kräftig genährt, danken für Alles lern',/ Und verstehe die Freiheit,/ Aufzubrechen, wohin er will. « ${ }^{66}$
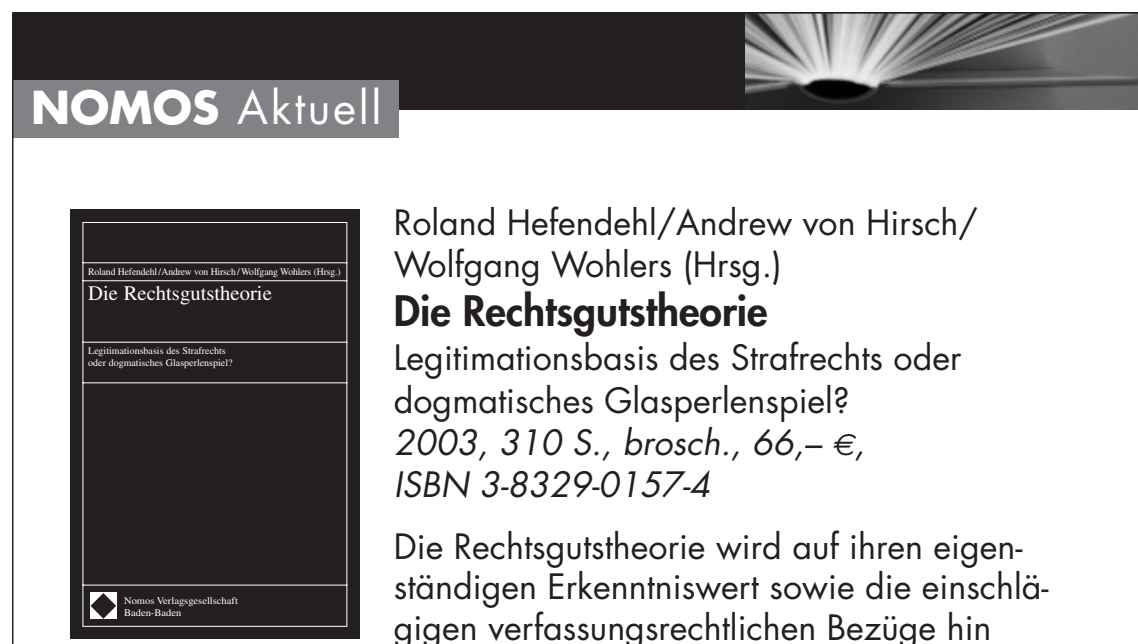

Roland Hefendehl/Andrew von Hirsch/

Wolfgang Wohlers (Hrsg.)

\section{Die Rechtsgutstheorie}

Legitimationsbasis des Strafrechts oder

dogmatisches Glasperlenspiel?

2003, 310 S., brosch., 66,-€,

ISBN 3-8329-0157-4

Die Rechtsgutstheorie wird auf ihren eigen-

ständigen Erkenntniswert sowie die einschlä-

gigen verfassungsrechtlichen Bezüge hin

untersucht. Weiterhin werden die Notwendig-

keit einer Ergänzung des Denkens in Rechts-

gütern durch Erwägungen zur Deliktsstruktur

herausgearbeitet sowie alternative Legitima-

tionsmodelle diskutiert.

NOMOS Verlagsgesellschaft • Baden-Baden
Baden-Baden F Fax 07221/2104-43 · nomos@nomos.de

65 In diesem Sinne auch : Nationaler Ethikrat, Stellungnahme: Genetische Diagnostik vor und während der Schwangerschaft, 2003, Mehrheitsvotum Nr. 9.5.

66 F. Hölderlin, Lebenslauf, 1798. 